\title{
Changes in Prescribed Treatments Following Parathyroidectomy for Primary Hyperparathyroidism in Older Adults
}

\author{
Shaun F Purkiss ${ }^{1}$, Tessa Keegel ${ }^{2}$, Hassan Vally ${ }^{3}$, Dennis Wollersheim ${ }^{4}$
}

\begin{abstract}
Aim and objective: Primary hyperparathyroidism (PHPT) is associated with multiple comorbidities and diverse symptomology that often require management with prescribed medications. Parathyroidectomy is an established treatment for PHPT but may also reduce the need for medications required to treat the associated conditions. We examined the changes in dispensed drugs given to a cohort of older adults with PHPT before and following parathyroidectomy.

Materials and methods: A publicly available administrative Pharmaceutical and Medical Benefits Scheme database was used for this study. Participants aged $>45$ years undergoing parathyroidectomy for PHPT were identified by procedure codes for the period 2008 to 2014 . Anatomic Therapeutic Chemical codes (ATC) assigned to drugs prescribed to individual participants, pre- and postoperatively, were used to define therapeutic groups and calculate cohort prevalences. Changes in dispensed medications following parathyroidectomy were assessed by the modulation of ATC class prevalence estimates of drugs prescribed using the Autoregressive Integrated Moving Averages (ARIMA) time-series methodology.

Results: Six hundred and ten persons aged $>45$ years (M 72.1, SD 7.0) undergoing parathyroidectomy for PHPT were recruited. Preoperative dispensed treatment prevalences were highest for agents acting on the renin-angiotensin system (48\%), dyslipidemia (45\%), acid disorders (37\%), antibiotics (31\%), and analgesics (20\%). Treatment prevalences for all ATC defined medications remained either unchanged or increased 3 years postoperatively. Polypharmacy following surgery also increased from an average 5.9 (SD =4.0, range $0-23$ ) prescribed medications for chronic conditions preoperatively to $7.0(\mathrm{SD}=4.0)$ at 2 years $(p<0.001)$.

Conclusion: The prevalence of ATC classified drugs prescribed and dispensed for chronic conditions in older adults with PHPT changed minimally following parathyroidectomy and polypharmacy of dispensed medications increased.

Clinical significance: Parathyroidectomy performed in older persons for PHPT has a limited impact on the prevalence of dispensed drugs used to treat many of the associated chronic conditions.

Mesh: Big data; Chronic conditions; Drug utilization; Hyperparathyroidism; Polypharmacy; Primary; Surgery.

Keywords: Administrative data, Chronic conditions, Medications, Parathyroidectomy, Polypharmacy, Primary hyperparathyroidism, Treatments. World Journal of Endocrine Surgery (2020): 10.5005/jp-journals-10002-1295
\end{abstract}

\section{InTRODUCTION}

Primary hyperparathyroidism (PHPT) is a common endocrine condition that can present in various ways. ${ }^{1}$ Many individuals with PHPT are diagnosed with routine biochemical analyzes. ${ }^{1-3}$ However, the presentation of PHPT is associated with chronic conditions, such as, peptic ulcer disease, cardiovascular disease, and osteoporosis. ${ }^{1}$ Primary hyperparathyroidism can also present with vague and nonspecific symptoms within gastrointestinal, neuropsychological, and musculoskeletal domains, all of which may require treatment using prescribed medications. ${ }^{4,5}$

Many of the clinical manifestations associated with PHPT as described can also be age-related. ${ }^{2}$ The peak incidence for PHPT occurs in the sixth decade with postmenopausal women affected most frequently. ${ }^{1,2}$ This has implications in longitudinal studies that assess interventions to treat PHPT. Parathyroidectomy will likely have a profound effect on calcium and parathyroid hormone levels but is less likely to alter the underlying impact of aging which will tend to increase the prevalence of most conditions requiring drug treatments. Appropriate time trend analyzes are therefore important to evaluate interventions, such as, parathyroidectomy for persons with PHPT to assess how the prevalence of medications required to treat the associated conditions may change.

\begin{abstract}
1,3,4 Department of Public Health, La Trobe University, Bundoora, Victoria, Australia

${ }^{2}$ Department of Public Health, La Trobe University, Bundoora, Victoria, Australia; Monash Centre for Occupational and Environmental Health, Monash University, Victoria, Australia
\end{abstract}

Corresponding Author: Shaun F Purkiss, Department of Public Health, La Trobe University, Bundoora, Victoria, Australia, Phone: +61 0426616025, e-mail: s.purkiss@latrobe.edu.au

How to cite this article: Purkiss SF, Keegel T, Vally H, et al. Changes in Prescribed Treatments Following Parathyroidectomy for Primary Hyperparathyroidism in Older Adults. World J Endoc Surg 2020;12(2):47-53.

Source of support: Nil

Conflict of interest: None

Parathyroidectomy is an established and effective treatment for PHPT with control of calcium and parathyroid hormone levels reported in $>95 \%$ of patients operated within specialist units. ${ }^{6}$ These biochemical improvements have also been considered to be associated with better control of symptoms and amelioration of comorbidities. ${ }^{7,8}$ Discontinuation and dose reduction of 
medications required for gastroesophageal reflux, bodily and bone pain, constipation, and psychological complaints have all been described following successful parathyroidectomy. ${ }^{4,7-9}$ From this perspective, some commentators have considered the presence of significant and potentially reversible comorbidity in patients with PHPT as an independent indication for surgical treatment. ${ }^{1,8,9}$ The therapeutic profile of patients before and after parathyroid surgery could therefore provide a perspective for research.

Individual patient medication can be tracked using prescription data. In Australia, these data are collected by the pharmaceutical benefits scheme (PBS) which provides universal access to necessary medicines for all Australians. Pharmaceutical Benefits Scheme prescription data has been collected over a long period and a sample of both the PBS data and the Medicare Benefits Schedule (MBS), detailing service items, was published in the public domain. ${ }^{10}$ This has provided an opportunity for health inquiry by linkage of the two data sets into many areas of population health including surgical outcomes.

Several chronic conditions and symptoms associated with PHPT can be implied by the treatments prescribed. ${ }^{11}$ Pain is characterized by the prescription of analgesics and other chronic conditions, such as, diabetes, for example, by other insulin and oral hypoglycemic treatments. Anatomic therapeutic chemical codes (ATC), developed by the World Health Organization (WHO) are an established system of therapeutic classification. ${ }^{12}$ Anatomic Therapeutic Chemical codes assigned to drugs can also be used for the case definition of persons with certain symptoms and chronic conditions. ${ }^{11}$ Systems using ATC methodologies have been developed for several healthrelated inquiries including insurance payments and populationlevel chronic disease prevalence estimates. ${ }^{11,13}$

In this study, a linked Australian PBS and medical service database were used and ATC defined treatments delineated for persons undergoing parathyroid surgery for PHPT. Changes in the dispensed treatment profiles were examined to assess the impact of surgery on this perspective.

\section{Materials and Methods}

\section{Study Design}

This study was a retrospective cohort study with a quasiexperimental design using linked administrative PBS and MBS data. ${ }^{10}$ Participants included persons over the age of 45 years undergoing parathyroidectomy for PHPT between 2008 and 2015. The profile of dispensed medications to participants was defined concerning the timing of surgery and assessed by examining changes in ATC treatment profiles.

\section{Participants}

Participants undergoing parathyroidectomy for PHPT were identified using MBS procedure codes from the MBS data set. Unique identifiers (UI) assigned to participants allowed data linkage from the MBS data set with the PBS data set to retrieve their individual medication histories. Participant demographic data collected at the time of the parathyroid surgery included age, sex, and the Australian state of origin.

\section{Data Sources}

The Australian Department of Health published a sample of the PBS and MBS databases publicly available under a Creative Commons Attribution 4.0 International License, and this was used for this study. ${ }^{10}$ These data are a collection of historical Medicare and PBS services relating to 2.2 million Australians. The data are linked using anonymized patient identifiers. The data are a random $10 \%$ sample of all patients within the PBS from 2003 to 2014 and MBS from 1993 to 2014.

\section{Data Storage and Confidentialization}

These data sets were retrieved and housed within a secure repository according to national digital information privacy standards. The data are de-identified at the source by the Australian Department of Health. The date of service was also randomly perturbed to within 14 days and the Medicare and PBS items with an extremely low-level volume of service were excluded.

\section{Outcome Variables}

The prevalence of specific ATC classes of treatments prescribed to participants undergoing parathyroidectomy for PHPT was calculated and used as the main variables examined in this study. Temporal changes in this dispensed treatment prevalence before and after parathyroid surgery were used to assess the potential impact of the surgery from this perspective. Anatomic Therapeutic Chemical codes were used to define a chronic condition or symptom for individual patients at quarterly time intervals pre- and postparathyroidectomy. An individual receiving a single ATC defined prescription in a defined quarter relative to the time of surgery was counted and aggregated for cohort prevalence estimates. The most prevalent conditions demarcated by ATC codes were defined and multimorbidity assessed by calculating the number of ATC defined groups of prescribed medications received by each individual. This number of multimorbidities was assessed at annual intervals for each person before and after parathyroidectomy and the total number of individuals in each multimorbidity grouping counted.

\section{Treatment Prevalence and Statistical Analysis}

Prevalence of medication usage, expressed as a proportion, was calculated for 12 quarters before and after surgery from the count of patients with at least one prescribed drug within a defined ATC group. The denominators for prevalence estimates were derived from the total number of potentially observable persons at each time. Pre- and postoperative prevalences of ATC treatment classes were represented graphically (Fig. 1) and assessment of an intervention effect utilized criteria that included a comparison of the averaged 3-year ATC defined treatment prevalence, before and after parathyroid surgery and identification of a change in treatment trends using Autoregressive Integrated Moving Averages (ARIMA) time-series analyzes. ${ }^{14} \mathrm{~A}$ template published by Cochrane was employed for the computation of ARIMA using models $(1,0,0)$ and $(1,1,0)$ in SPSS. ${ }^{14}$ Treatment prevalence trends were also assessed for stationarity using the Dickey-Fuller test $(p>0.05)$ performed in RStudio. ${ }^{15}$ Comparison of participant's gender, average ages, treatment prevalence utilized the two-sample and paired students $t$-tests and one-way ANOVA for comparison between states. A comparison of multimorbidity status used the Wilcoxon signed-rank test. Statistical significance was set at $p<0.05$. The presentation of this study follows the guidelines recommended in the REporting of studies conducted using observational routinely-collected health data (RECORD) statement. ${ }^{16}$

\section{Results}

Six hundred and ten participants undergoing parathyroidectomy for PHPT between 2008 and 2014 were recruited into this study. Seven 


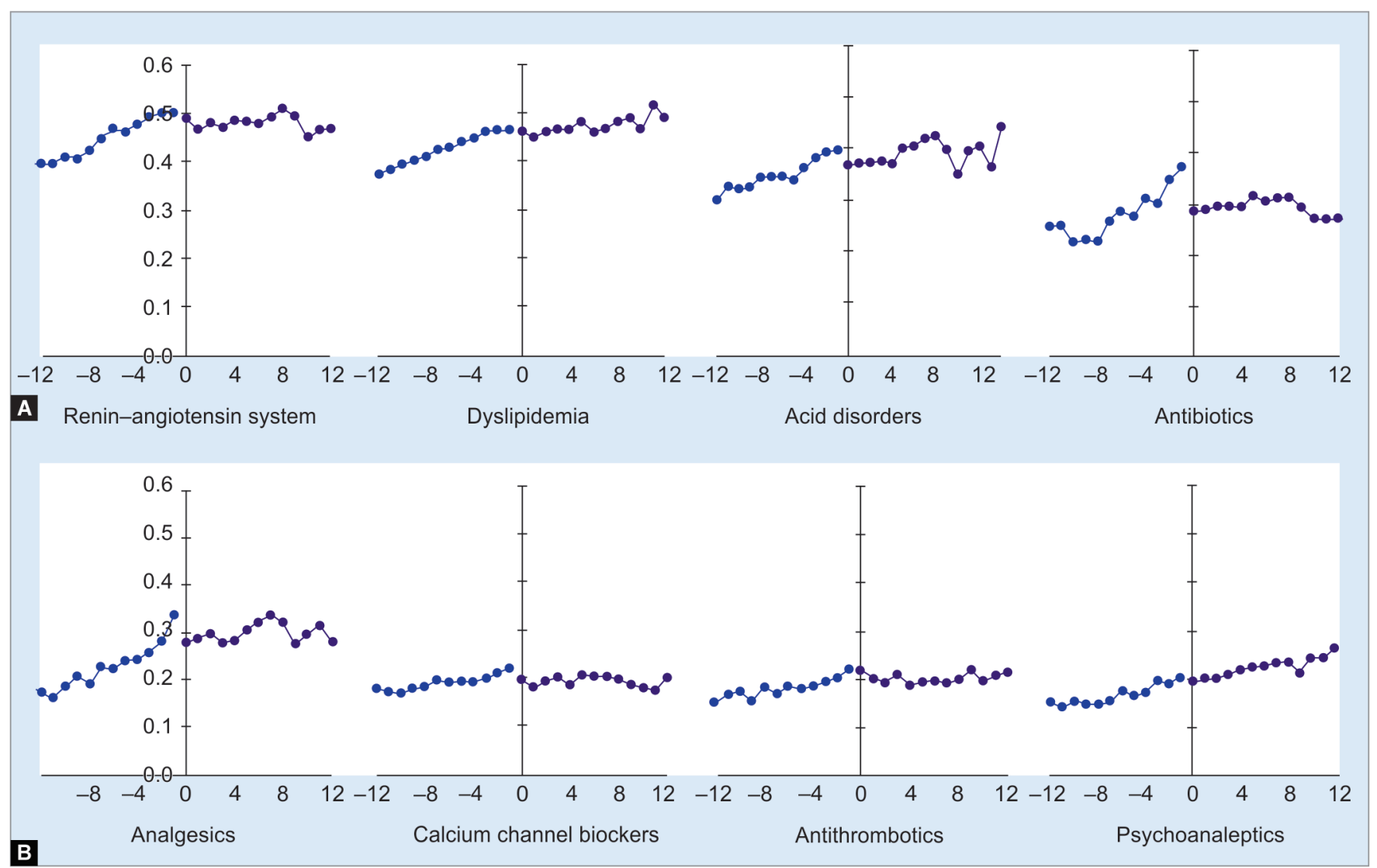

Figs $1 \mathrm{~A}$ and B: Pre- and postoperative, 6-year (24 quarters) treatment prevalences of ATC defined conditions, described as a proportion (0.0-0.6), for persons undergoing parathyroidectomy for PHPT at time zero between 2008 and 2015

Table 1: Average age (SD) and the count of participants undergoing parathyroidectomy for primary hyperparathyroidism from Australian states for the period 2008 to 2015

\begin{tabular}{|c|c|c|c|c|c|c|}
\hline \multirow[b]{2}{*}{ Year } & \multicolumn{5}{|c|}{ Australian state of origin } & \multirow[b]{2}{*}{ Total } \\
\hline & NSW & QLD & $S A$ & VIC & $W A$ & \\
\hline Persons 2008-2015 & 272 & 95 & 58 & 127 & 58 & 610 \\
\hline F/M counts & $205 / 67$ & $69 / 26$ & $45 / 13$ & $100 / 27$ & $44 / 14$ & $463 / 147$ \\
\hline 2008 & 30 & 9 & 6 & 9 & 3 & 57 \\
\hline 2009 & 33 & 13 & 6 & 13 & 5 & 70 \\
\hline 2010 & 37 & 13 & 5 & 22 & 10 & 87 \\
\hline 2011 & 28 & 17 & 13 & 20 & 9 & 87 \\
\hline 2012 & 46 & 12 & 14 & 17 & 11 & 100 \\
\hline 2013 & 47 & 12 & 6 & 23 & 9 & 97 \\
\hline 2014 & 51 & 19 & 8 & 23 & 11 & 112 \\
\hline Average age & 72.5 & 71.7 & 70.3 & 72.4 & 72.3 & 72.1 \\
\hline (SD) & 8.6 & 14.4 & 18.1 & 12.6 & 18.6 & 15.2 \\
\hline
\end{tabular}

years pre- and postoperative observations of cohort treatment prevalences were analyzed. Following parathyroidectomy, the number of participants observed within the cohort was 610 at 4 months and reduced to 121 persons at 40 months. This reduction occurred as the consequence of persons having their parathyroidectomy toward the end of the data-set sample period. These individuals had progressively shorter postoperative surveillance periods, thus gradually reducing the number of participants with a complete 40 months of observations after surgery. The demographic variables of participants retrieved at the time of surgery are shown in Table 1. The average age at parathyroidectomy was $72.1(\mathrm{SD}=7.0)$ years. There were no significant differences in average age at operation between males and females $(p=0.18)$ and between the Australian states $(p=0.29)$.

\section{Treatment Prevalence}

The treatments with a higher prevalence in preoperative persons undergoing parathyroidectomy for PHPT are shown in Figure 1 and Table 2. The ATC codification associated with these morbidities is also shown within the table. The most prevalent 
Table 2: ATC defined dispensed treatment prevalences before and parathyroid surgery for PHPT, combined with stationarity, ARIMA analyzes, and assessment of the overall effect

\begin{tabular}{|c|c|c|c|c|c|c|c|c|}
\hline \multirow{2}{*}{$\begin{array}{l}\text { Therapeutic class } \\
\text { Description }\end{array}$} & \multirow[b]{2}{*}{ ATC code } & \multicolumn{2}{|c|}{ Prevalence Mean, (SD) } & \multirow[b]{2}{*}{$\begin{array}{l}\text { Significance } \\
(<0.05)\end{array}$} & \multirow{2}{*}{$\begin{array}{l}\text { Stationarity } \\
\text { Pre/post } \\
\text { change }\end{array}$} & \multicolumn{2}{|c|}{ ARIMA result } & \multirow{2}{*}{$\begin{array}{l}\text { Assessment } \\
\begin{array}{l}\text { Treatment } \\
\text { effect }\end{array}\end{array}$} \\
\hline & & Pre 18 months & $\begin{array}{l}\text { Post } 36 \\
\text { months }\end{array}$ & & & $\begin{array}{l}\text { Prevalence } \\
\text { change }\end{array}$ & $\begin{array}{l}\text { Significance } \\
(<0.05)\end{array}$ & \\
\hline $\begin{array}{l}\text { Renin-angiotensin } \\
\text { agents }\end{array}$ & C09 & $0.48(0.02)$ & $0.46(0.01)$ & $n s^{b}$ & $\mathrm{n} / \mathrm{y}^{* * \mathrm{c}}$ & nil & ns & No effect \\
\hline Dyslipidemia & C10 & $0.45(0.02)$ & $0.47(0.01)$ & $p<0.001 \uparrow^{d}$ & $n / y^{* *}$ & nil & ns & No effect \\
\hline Acid disorders & $\mathrm{A} 02$ & $0.37(0.02)$ & $0.39(0.01)$ & $p<0.001 \uparrow$ & $\mathrm{n} / \mathrm{n}$ & nil & ns & No effect \\
\hline Antibiotics & J01 & $0.31(0.04)$ & $0.30(0.01)$ & ns & $n / y^{* *}$ & $0.059 \downarrow^{\mathrm{e}}$ & 0.005 & Yes \\
\hline Analgesics & N02 & $0.27(0.04)$ & $0.31(0.01)$ & ns & $\mathrm{n} / \mathrm{y}^{* *}$ & nil & ns & No effect \\
\hline $\begin{array}{l}\text { Calcium channel } \\
\text { blockers }\end{array}$ & $\mathrm{C} 08$ & $0.20(0.01)$ & $0.19(0.01)$ & ns & $n / y^{* *}$ & nil & ns & No effect \\
\hline Psychoanaleptics & N06 & $0.19(0.02)$ & $0.22(0.01)$ & $p<0.001 \uparrow$ & $\mathrm{n} / \mathrm{n}$ & nil & ns & No effect \\
\hline Beta-blockers & $\mathrm{CO} 7$ & $0.17(0.01)$ & $0.19(0.01)$ & $p<0.001 \uparrow$ & $\mathrm{n} / \mathrm{n}$ & nil & ns & No effect \\
\hline Ophthalmologicals & S01 & $0.15(0.01)$ & $0.20(0.01)$ & $p<0.001 \uparrow$ & $n / n$ & nil & ns & Increased use \\
\hline $\begin{array}{l}\text { Drugs used in bone } \\
\text { diseases }\end{array}$ & M05 & $0.14(0.01)$ & $0.15(0.01)$ & ns & $y / y$ & nil & ns & No effect \\
\hline Antirheumatics & M01 & $0.14(0.01)$ & $0.14(0.01)$ & ns & $y / y$ & nil & ns & No effect \\
\hline COPD and asthma & R03 & $0.12(0.01)$ & $0.17(0.01)$ & $p<0.001 \uparrow$ & $y / n$ & $0.004 \uparrow$ & 0.013 & No effect \\
\hline Psycholeptics & N05 & $0.10(0.01)$ & $0.15(0.02)$ & $p<0.001 \uparrow$ & $y / y$ & nil & ns & Increased use \\
\hline Drugs used in diabetes & A10 & $0.08(0.01)$ & $0.11(<0.01)$ & $p<0.001 \uparrow$ & $n / n$ & nil & ns & No effect \\
\hline $\begin{array}{l}\text { Antihypertensive } \\
\text { agents }\end{array}$ & $\mathrm{CO2}$ & $0.05(0.01)$ & $0.03(<0.01)$ & ns & $\mathrm{n} / \mathrm{n}$ & $-0.002 \downarrow$ & 0.013 & No effect \\
\hline Antianemic agents & B03 & $0.05(0.01)$ & $0.06(0.01)$ & $p=0.024 \uparrow$ & $y / y$ & nil & ns & No effect \\
\hline $\begin{array}{l}\text { Drugs used in } \\
\text { constipation }\end{array}$ & A06 & $0.04(0.01)$ & $0.09(0.01)$ & $p<0.001 \uparrow$ & $n / n$ & $0.003 \uparrow$ & 0.012 & Increased use \\
\hline Calcium agents & A12 & $0.02(0.01)$ & $0.03(0.01)$ & $p<0.001 \uparrow$ & $y / y$ & nil & ns & No effect \\
\hline Vitamin D & A11 & $0.01(<0.01)$ & 0.03 & $p<0.001 \uparrow$ & $y / n^{* *}$ & $-0.004 \downarrow$ & 0.011 & Increased use \\
\hline
\end{tabular}

${ }^{a}$ Change in stationarity assessed using Dickey-Fuller test $(p>0.05)$

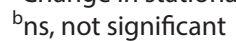

c**change in prevalence stationarity

${ }_{1}$, Increase in prevalence postoperatively

$e_{\downarrow}$, Decrease in prevalence postoperatively

treatments identified included therapies using agents acting on the renin-angiotensin system, dyslipidemia, acid disorders, and other treatments for cardiovascular disease and hypertension. The prevalence of treatments preoperatively for bodily pain with opiate and non-opiate analgesia was 0.10 and 0.18 , respectively. Anti-inflammatory agents were prescribed at prevalence levels of 0.14 , and agents used in bone disease 0.15 . Treatments prescribed for functional bowel disorders, constipation, and antidiarrheal were prescribed at lower prevalence levels of $<0.05$. Antibiotic treatments, in contrast, were prescribed at increasing prevalence levels during the period before parathyroidectomy, reaching estimates of 0.37 in the immediate preoperative quarter.

\section{Prevalence Modulation}

The impact of parathyroidectomy as a health intervention for PHPT and determined by the changes in the pre- and postoperative prevalence trends of selected dispensed treatments are shown in Figure 1 and Table 2. Treatment prevalences showed minimal change for most conditions. The trends of prevalence for treatments for glaucoma, digitalis medication, beta-blockers, anxiolytics, diabetes, gout, antidepressants, and antacid medications were all unaffected by parathyroid surgery. The prevalence of these conditions demonstrated non-stationarity and tended to rise at varying degrees over both the pre- and postoperative periods of observations (Fig. 1). Overall, treatment prevalence rose significantly between the immediate preoperative and postoperative periods in 8 of 29 assessed ATC categories. The remaining ATC categories showed no significant changes to preoperative prevalence levels (Table 1).

Applying the three criteria of treatment prevalence pre- and postoperatively, change in the stationarity of prevalence and ARIMA assessment of prevalence to assess the impact of parathyroidectomy, 24 treatment prevalences were unaffected by surgery (Table 2). Three conditions (ophthalmological, psycholeptics, and drugs used in constipation) had an increase in treatment prevalence after surgery, and one treatment, antibiotic usage, showed a beneficial early intervention effect (Fig. 1 and Table 2).

\section{Multimorbidity}

The average number of ATC groups prescribed in the preoperative year before parathyroidectomy was $5.9(S D=4.0)$, with a range of 0-23 different treatments (Fig. 2). Two years postoperatively, polypharmacy was significantly higher with a mean of 7.0 (SD 4.0) treatments $(p<0.001)$. The changes in the number of ATC defined multimorbidities at 2 years postoperatively varied. Those persons with 0-9 ATC distinct treatments prescribed preoperatively had 
significantly more treatment categories prescribed at 2 years postoperatively ( $p<0.05$ ) (Fig. 3). Whereas those prescribed between 10 and 15 ATC grouped treatments remained at similar levels 2 years postoperatively. In contrast, persons with $>16$ ATC distinct prescribed treatments at the time of parathyroidectomy tended to have fewer prescribed at 2 years. A similar finding was noticed with prescribed treatments for cardiovascular disorders and hypertension (ATC codes C01-09) (Fig. 3). Overall, dispensed treatments within these groups of cardiovascular drugs rose from 2.72 ATC defined groups preoperatively to 2.84 at 2 years postoperatively $(p<0.001)$. This finding was influenced predominantly by persons receiving less than two cardiovascular drugs preoperatively who subsequently had more treatments prescribed at 2 years. Persons receiving higher levels of cardiovascular polypharmacy had no significant change in the number of cardiovascular treatments dispensed over the same durations.

\section{Discussion}

This study describes a novel approach using cohort treatment prevalence calculated from the Australian administrative pharmaceutical data to assess the impact of parathyroid surgery for persons with PHPT. This approach provides a perspective that potentially avoids the biases present in surveys related to participation and self-reported phenomena. ${ }^{17}$ Furthermore, research using administrative data has been recognized as superior to respondent surveys concerning drugs received by patients in many healthcare domains. ${ }^{18}$ As a consequence, offsetting the effect of recall bias by study participants is a strength of this study examining the cohort prevalence of dispensed prescribed medications. Administrative pharmaceutical database research can also provide new perspectives to many aspects of health care and has been used previously for the evaluation of outcomes in cardiac and orthopedic surgery, and surgically related nosocomial infection. ${ }^{19-21}$

A benefit of the approach used in this study is the scope for longitudinal analysis with a quasi-experimental design. This has enabled the use of medication profiles of persons undergoing parathyroidectomy for PHPT to be analyzed pre- and postoperatively using 7 years of data. Previous studies exploring therapeutic improvements after parathyroid surgery have used shorter perioperative windows of inquiry and not controlled for

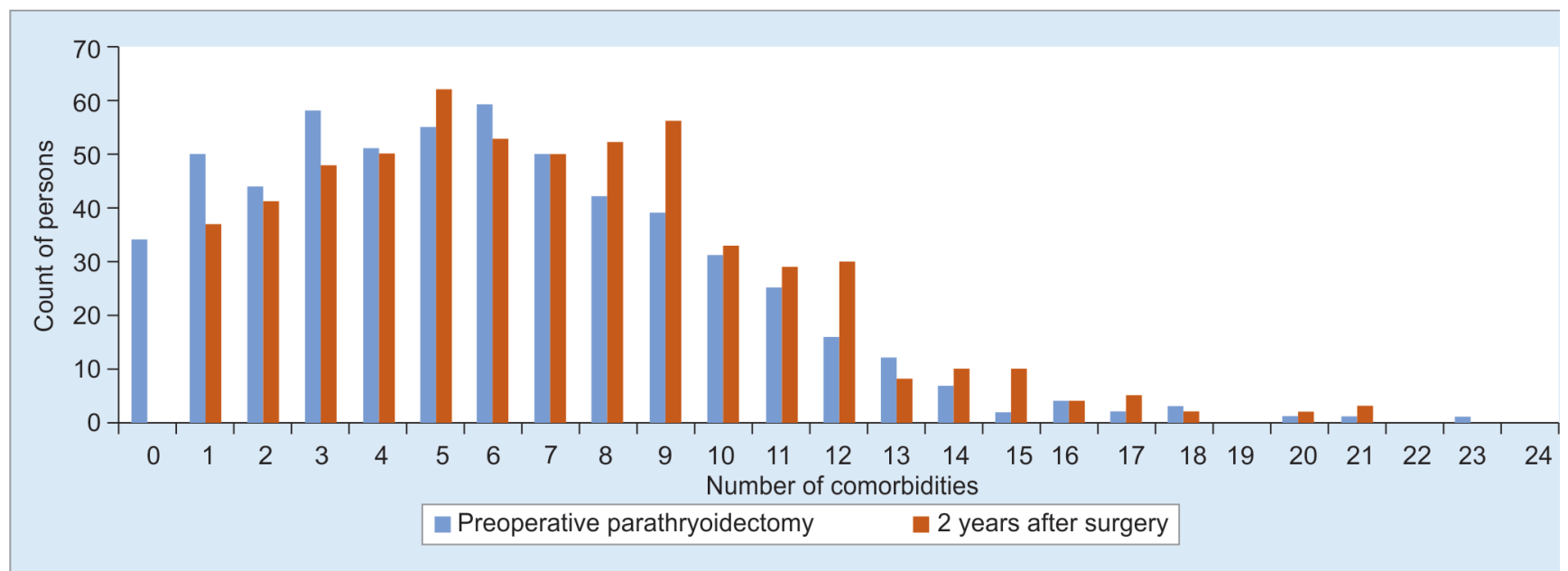

Fig. 2: Number of persons undergoing parathyroidectomy for PHPT with the sums of persons having a range of multiple morbidities (range 0-23) at the time of surgery and 2 years postoperatively
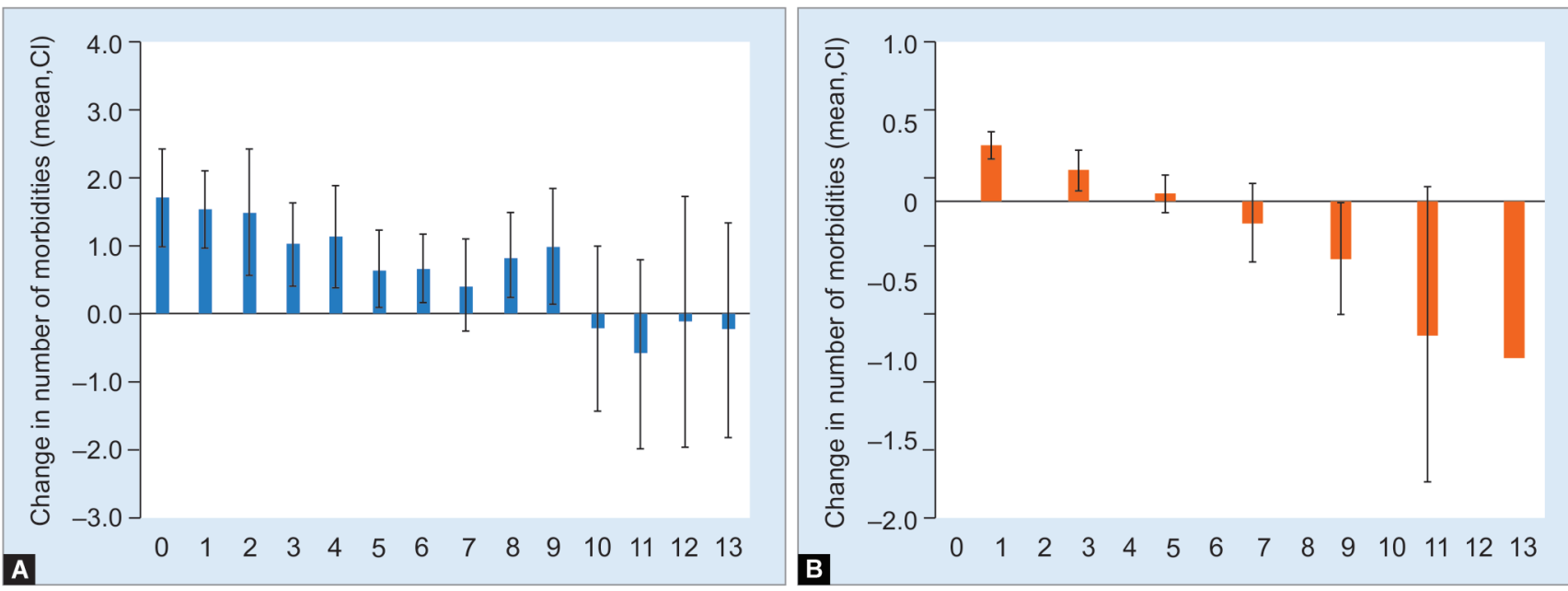

Figs $3 \mathrm{~A}$ and B: Changes in multimorbidity at 2 years postoperatively following parathyroidectomy for PHP for (A), all ATC defined groups for (B), cardiac medications 
increasing age as a confounding covariate. ${ }^{7-9}$ In addition, previous reports have employed static statistical tests, which are recognized in time-series analyzes to produce results that can be inaccurate, and of limited detail. ${ }^{8}$ Time-series analyzes as used in this study with ARIMA methodology can evaluate trends and use all pre- and postoperative prescription data to assess treatment effects. This is in contrast to other studies that used single prevalence measures at set points before and after surgery to assess the impact of surgery. ${ }^{14}$ This is important where the confounding effect of increasing age may in general increase the prevalence of most chronic conditions associated with PHPT, thus masking any potential effect of parathyroidectomy. Accordingly, the results of this study using treatment prevalence estimates, trend stationarity, and ARIMA methodology to control for age-related effects, suggest that the impact of parathyroidectomy upon the dispensed treatments prescribed for conditions associated with PHPT is constrained.

The potential limitations of this study include the data source and cohort selection. The data are limited by the restricted number of covariates available for analysis. This is recognized as a general limitation with administrative data when used for other purposes. ${ }^{18}$ Hence, the demographic data retrieved in this study are restricted to participant age, gender, and state of origin. As a consequence, the extent of detail that can be obtained from survey responses, for example, is not possible with administrative data. ${ }^{18}$ This study is also reliant on accurate codification for the case definition of persons undergoing parathyroidectomy. The potential for misclassifications of procedure codes is acknowledged and may apply in distinguishing between persons undergoing parathyroidectomy for either PHPT or secondary hyperparathyroidism. ${ }^{22}$ However, the cohort profile of the long-term survival of the participants in this study is similar to other Australian data for patients with PHPT. ${ }^{23,24}$ The cohort examined in this study were older adults undergoing parathyroid surgery after 2008 and is a recognized subgroup suitable for assessment. ${ }^{22}$ This group was selected because of the time frame of the data and recognition that elderly persons are more likely to utilize prescription services at higher levels than younger groups. ${ }^{25}$ The potential for this to limit the generalizability of these findings to younger persons with less risk of chronic disease is acknowledged. This group could well have better symptom improvement following parathyroidectomy for PHPT than those demonstrated in this study with an older cohort. Furthermore, it is recognized that parathyroidectomy for PHPT is associated with a variety of other healthcare inputs which may also influence outcome., ${ }^{1,26}$ The effects of these interventions connected with the multidisciplinary management of persons with PHPT cannot be distinguished from the surgical procedure in this study. The study assumes that those interventions and the quality of the surgery are consistent between participants.

Primary hyperparathyroidism is associated with a wide variety of comorbidities and symptoms that can influence the welfare and presentation of persons with this condition. ${ }^{3,26}$ Parathyroidectomy has established success in PHPT for the control of hypercalcemia and parathyroid hormone levels, which have also been considered to be the underlying components that cause symptoms. ${ }^{1,2}$ On this premise, benefits in the management of associated conditions following parathyroidectomy for PHPT have been reported. Roman and Sosa commented that psychiatric and neurocognitive functioning was better after parathyroidectomy and Norman et al. recorded the amelioration of gastroesophageal reflux symptoms following successful surgery. ${ }^{4,7}$ In both these studies, the authors considered the improvements were of such a significant and durable magnitude that their presence preoperatively should be considered independent indicators for the consideration of surgery. ${ }^{4,7}$ In more global assessments of well-being, Pasieka and Parsons quantified improved quality of life scores, and Melck et al. identified symptom improvements and medication changes in several domains after successful parathyroidectomy. ${ }^{8,9}$ The therapeutic changes included reductions in analgesic requirements, antacids, antidepressants, anxiolytics, and stool softeners. ${ }^{8}$ The results of this study provide contrast to these previous reports by indicating the limited efficacy of parathyroidectomy in modulating most treatment trends in older adults with PHPT. Opiate and non-opiate analgesic use in addition were specifically noted to be unaffected by parathyroidectomy.

The prevalence of vitamin D treatments and calcium supplements increased following parathyroidectomy which may be related to the metabolic consequences of the surgery, but in general, these treatments were prescribed at low levels. Vitamin $\mathrm{D}$ treatment prevalence was 0.08 immediately after surgery and reduced gradually thereafter and calcium supplementations varied between 0.02 and 0.05 postoperatively. In an early study, Westerdahl et al. reported hypocalcemia following parathyroidectomy for PHPT in $25.6 \%$ of patients in the early phase, reducing to $18.6 \%$ at 1 year. ${ }^{27}$ These are magnitudes of differences from the values reported in this study that might be related to the availability of these treatments over the counter without the need for prescription or could also be related to the introduction of minimally invasive parathyroidectomy by the Australian surgeons. This technique may avoid some of the intraoperative trauma known to be associated with the risk of developing postoperative hypocalcemia. ${ }^{28}$

This study has provided longitudinal perioperative treatment profiles for a cohort of older adults undergoing parathyroidectomy for PHPT. The study used a quasi-experimental approach to assess the effects of surgery and employed an Australian pharmaceutical database linked to a service provision (MBS) data set identifying surgical procedures. Time-series analysis of ATC-defined prescribed drugs showed limited efficacy of parathyroidectomy for persons with PHPT to alter the prevalence of dispensed treatments used to manage their associated comorbid conditions.

\section{Clinical Significance}

These findings suggest that in older persons, parathyroidectomy has a limited impact on the prevalence of dispensed drugs used to treat many of the chronic conditions associated with PHPT.

\section{Acknowledgments}

The authors acknowledge the La Trobe ethics committee who granted ethical approval for this study and Dr Xia Li for providing statistical advice.

\section{References}

1. Khan AA, Hanley DA, Rizzoli R, et al. Primary hyperparathyroidism: review and recommendations on evaluation, diagnosis, and management. A Canadian and international consensus. Osteoporos Int 2017;28(1):1-9. DOI: 10.1007/s00198-016-3716-2.

2. Silverberg SJ, Shane E, Jacobs TP, et al. A 10-year prospective study of primary hyperparathyroidism with or without parathyroid surgery. N Eng J Med 1999;341(17):1249-1255. DOI: 10.1056/ NEJM199910213411701.

3. Silverberg SJ, Clarke BL, Peacock M, et al. Current issues in the presentation of asymptomatic primary hyperparathyroidism: 
proceedings of the fourth international workshop. J Clin Endocrinol Metabol 2014;99(10):3580-3594. DOI: 10.1210/jc.20141415.

4. Roman S, Sosa JA. Psychiatric and cognitive aspects of primary hyperparathyroidism. Curr Opin Oncol 2007;19(1):1-5. DOI: 10.1097/ CCO.0b013e32801173fb.

5. Rubin MR, Bilezikian JP, McMahon DJ, et al. The natural history of primary hyperparathyroidism with or without parathyroid surgery after 15 years. J Clin Endocrinol Metabol 2008;93(9):3462-3470. DOI: 10.1210/jc.2007-1215.

6. Clark $\mathrm{OH}$. Surgical treatment of primary hyperparathyroidism. Adv Endocrinol Metab 1995;6:1-6.

7. Norman J, Politz D, Lopez J, et al. Surgical cure of primary hyperparathyroidism ameliorates gastroesophageal reflux symptoms. World J Surg 2015;39(3):706-712. DOI: 10.1007/s00268014-2876-5.

8. Melck AL, Armstrong MJ, Stang MT, et al. Medication discontinuation after curative surgery for sporadic primary hyperparathyroidism. Surgery 2010;148(6):1113-1119. DOI: 10.1016/j.surg.2010.09.008.

9. Pasieka JL, Parsons LL. Prospective surgical outcome study of relief of symptoms following surgery in patients with primary hyperparathyroidism. World J Surg 1998;22(6):513-519. DOI: 10.1007/ s002689900428.

10. Mellish L, Karanges EA, Litchfield MJ, et al. The Australian pharmaceutical benefits scheme data collection: a practical guide for researchers. BMC Res Notes 2015;8(1):634. DOI: 10.1186/s13104015-1616-8.

11. Huber CA, Diem P, Schwenkglenks M, et al. Estimating the prevalence of comorbid conditions and their effect on health care costs in patients with diabetes mellitus in Switzerland. Diabetes Metab Syndr Obes 2014;7:455. DOI: 10.2147/DMSO.S69520.

12. Miller GC, Britt H. A new drug classification for computer systems: the ATC extension code. Int J Biomed Comput 1995;40(2):121-124. DOI: 10.1016/0020-7101(95)01135-2.

13. Lamers LM, Vliet RC. Health-based risk adjustment Improving the pharmacy-based cost group model to reduce gaming possibilities. Eur J Health Econom 2003;4(2):107-114. DOI: 10.1007/s10198-0020159-9.

14. Cochrane Effective Practice and Organisation of Care (EPOC). Interrupted time series (ITS) analyses. EPOC Resources for review authors, 2017. Available at: http://epoc.cochrane.org/epoc-specificresources-review-authors.

15. Dickey DA, Fuller WA. Distribution of the estimators for autoregressive time series with a unit root. J Am Stat Assoc 1979;74(366a):427-431. DOI: 10.1080/01621459.1979.10482531.

16. Benchimol El, Smeeth L, Guttmann A, et al. The reporting of studies conducted using observational routinely-collected health data
(RECORD) statement. PLoS Med 2015;12(10):e1001885. DOI: 10.1371/ journal.pmed.1001885.

17. Harvey SB, Deady $M$, Wang MJ, et al. Is the prevalence of mental illness increasing in Australia? evidence from national health surveys and administrative data, 2001-2014. Med J Aust 2017;206(11):490-493. DOI: 10.5694/mja16.00295.

18. Schneeweiss S, Avorn J. A review of uses of health care utilization databases for epidemiologic research on therapeutics. J Clin Epidemiol 2005;58(4):323-337. DOI: 10.1016/j.jclinepi.2004.10.012.

19. Al-Jaishi AA, Moist LM, Oliver MJ, et al. Validity of administrative database code algorithms to identify vascular access placement, surgical revisions, and secondary patency. J Vasc Access 2018;19(6):561-568. DOI: 10.1177/1129729818762008.

20. Abdul-Jabbar A, Takemoto S, Weber MH, et al. Surgical site infection in spinal surgery: description of surgical and patient-based risk factors for postoperative infection using administrative claims data. Spine 2012;37(15):1340-1345. DOI: 10.1097/BRS.0b013e318246a53a.

21. Thomas C, Cadwallader HL, Riley TV. Surgical-site infections after orthopaedic surgery: statewide surveillance using linked administrative databases. J Hospital Infect 2004;57(1):25-30. DOI: 10.1016/j.jhin.2004.01.025.

22. Funk MJ, Landi SN. Misclassification in administrative claims data: quantifying the impact on treatment effect estimates. Curr Epidemiol Rep 2014;1(4):175-185. DOI: 10.1007/s40471-014-0027-z.

23. Purkiss S, Keegal T, Wollersheim D, et al. The efficacy of surgical parathyroidectomy assessed using Australian administrative pharmaceutical data and quasi-experimental design. Int J Populat Data Sci 2018(2):3. DOI: 10.23889/ijpds.v3i2.573.

24. Clifton-Bligh PB, Nery ML, Supramaniam R, et al. Mortality associated with primary hyperparathyroidism. Bone 2015;74:121-124. DOI: 10.1016/j.bone.2014.12.067.

25. Denizot A, Grino M, Oliver C. Surgical management of primary hyperparathyroidism in older adults. J Am Geriatr Soc 2014;62(9):17591763. DOI: 10.1111/jgs.12968.

26. Shindo M, Lee JA, Lubitz CC, et al. The changing landscape of primary, secondary, and tertiary hyperparathyroidism: highlights from the American College of Surgeons Panel,"What's new for the surgeon caring for patients with hyperparathyroidism". J Am Coll Surg 2016;222(6):1240-1250. DOI: 10.1016/j.jamcollsurg.2016.02.024.

27. Westerdahl J, Lindblom P, Valdemarsson S, et al. Risk factors for postoperative hypocalcemia after surgery for primary hyperparathyroidism. Arch Surg 2000;135(2):142-147. DOI: 10.1001/ archsurg.135.2.142.

28. Reilly DJ, Chew GL, Eckhaus J, et al. Outcomes for minimally invasive parathyroidectomy: widening inclusion criteria based on preoperative imaging results. ANZ J Surg 2016;86(9):701-705. DOI: 10.1111/ans.12885. 